\title{
ON THE EXTREMALITY AND UNIQUE EXTREMALITY OF AFFINE MAPPINGS IN SPACE, AN ADDENDUM
}

\author{
STEPHEN AGARD and RICHARD FEHLMANN
}

The proper use of the number $\exp \sqrt{n / 6(n-1)}$ in our above mentioned paper ([1]) depended on the crucial inequality $\psi \geqq 2 / 3$ whenever $\varphi=0$. There were Cases (II) and (III). In Case (II), $\varphi$ and $\psi$ were the following functions of the two variables $\alpha, \beta: \psi=F /(n+1) ; \varphi=\alpha^{k-1} \beta^{m-1} G, \quad$ in which $F(\alpha, \beta)=k(\alpha-1)^{2}+k m(\alpha-\beta)^{2}+$ $m(\beta-1)^{2} ; \quad G(\alpha, \beta)=k \beta(\alpha-1)^{2}+k m(\alpha-\beta)^{2}+m \alpha(\beta-1)^{2}$. Here $k, m, n$ are positive integers with $k+m=n$ and $n \geqq 2$. The case $\alpha=\beta=1$ is an irrelevant exception to the inequality. Aithough the case $\alpha=0$ had been treated, we neglected to treat the vanishing of $G$. It is the purpose of this note to correct this oversight for both this and the companion Case (III).

To begin, and by symmetry, we may assume that $\alpha \leqq \beta$, hence $G=0$ can only occur if $\alpha \leqq 0$. If $\beta<0$, then $F>k+m=n \geqq(2 / 3)(n+1)$. If $\beta-\alpha>1$, then $F>k+k m=k(n+1-k) \geqq n \geqq(2 / 3)(n+1)$. This could restrict consideration to the triangle $T=\{(a, \beta): 0 \leqq \beta \leqq \alpha+1,-1 \leqq \alpha \leqq 0\}$, but we prefer to consider the square $Q=\{(\alpha, \beta):-1 \leqq \alpha \leqq 0,0 \leqq \beta \leqq 1\}$, in which $F$ assumes its absolute minimum at $(\alpha, \beta)=(0,1 /(k+1))$, having there the value $(n+1) k /(k+1)$. This in turn is not less than $(2 / 3)(n+1)$ as soon as $k \geqq 2$. Hence all cases $k \geqq 2$ are done.

In case $k=1$, and on any vertical line (fixed $\alpha$ ) which meets $Q, G$ is a quadratic function of $\beta$, and since $G(\alpha, 0)=(n-1)\left(\alpha+\alpha^{2}\right) \leqq 0<n(\alpha-1)^{2}=G(\alpha, 1)$, it follows that the set $G=0$ meets each vertical section of $Q$ in exactly one point.

We define the pair $(B, M)$ by saying that the level curve $\{F=(2 / 3)(n+1)\}$ (an ellipse) has its lower meeting with the $\beta$-axis when $\beta=B$, having there slope $M$. The tangent line $L: \beta=M \alpha+B$, which is a lower support line for the sublevel set $K=\{F<(2 / 3)(n+1)\}$, enters $Q$ from the right at $(0, B)$, and crosses the line $\alpha=-1$ at $\beta=B-M$. As we will soon see, this number is $1-2 B \in(0,1]$. Our objective will now be to show that $G$ is nonnegative on $L$. It will then follow that the locus $G=0$ has no contact with $K$.

The defining conditions are: $B$ is the smaller root of $\beta^{2}+(\beta-1)^{2}=$ $(2 n-1) / 3(n-1)$, whereas $M=-F_{\alpha}(0, B) / F_{\beta}(0, B)=[(n-1) B+1] /(n-1)(2 B-1)$. More explicitly, the formula $B=(1 / 2)(1-\sqrt{(n+1) / 3(n-1)})$ shows that $B$ increases from 0 to $(1 / 2)(1-\sqrt{1 / 3})<0.22$ as $n$ runs from 2 to $\infty$. In particular, $1-2 B \in(0,1]$. The equally crucial and easily checked relation $M=3 B-1$ is helpful in simplifying 
the following expressions, where we write $g(\alpha)=G(\alpha, M \alpha+B)=A_{0}+A_{1} \alpha+A_{2} \alpha^{2}+$ $A_{3} \alpha^{3}$, and in which

$$
\begin{aligned}
& A_{0}=B+(n-1) B^{2}=n B-(n-2) / 6=1 / 3+n[B-(1 / 6)] \geqq 0, \\
& A_{1}=M-2 B+(n-1)\left[2 B(M-1)+(B-1)^{2}\right]=A_{0}, \\
& A_{2}=B-2 M+(n-1)\left[2 M(B-1)+(M-1)^{2}\right]=1+(1 / 2) n(7-10 B), \\
& A_{3}=M+(n-1) M^{2}=3 n B-(1 / 2)(n-2)=1+(1 / 2) n(-1+6 B)=3 A_{0} \geqq 0 .
\end{aligned}
$$

Regarding the claim $A_{0} \geqq 0$ : one sees $B=1 / 6$ when $n=7$. The case $n=2$ giving $A_{0}=0$, the remaining cases $n=3,4,5,6$ are checked individually. We now see in addition that $A_{2}-A_{3}=4 n(1-2 B)>0$. We deduce from the variously displayed relations:

$$
\begin{aligned}
g(\alpha) & =A_{0}+A_{1} \alpha+A_{2} \alpha^{2}+A_{3} \alpha^{3}=A_{0}\left(1+\alpha+3 \alpha^{2}+3 \alpha^{3}\right)+4 n(1-2 B) \alpha^{2} \\
& \geqq A_{0}\left(1+\alpha+3 \alpha^{2}+3 \alpha^{3}\right)=A_{0}(1+\alpha)\left(1+3 \alpha^{2}\right) \geqq 0,
\end{aligned}
$$

whenever $-1 \leqq \alpha \leqq 0$, with equality only if $\alpha=0$ and $A_{0}=0 \quad(n=2)$.

Turning to Case (III), the analogue to $(n+1) \psi=F$ may be expressed by $x A \cdot x-2 x \cdot v+n$, in which $x$ is the row vector $(\alpha, \beta, \gamma), v$ is the row vector $(k, m, p)$, and as before $k, m, p, n$ are positive integers with $k+m+p=n$. Here, $A$, and for later use, $E$, are the matrices:

$$
A=\left[\begin{array}{ccc}
k(n+1-k) & -m k & -k p \\
-m k & m(n+1-m) & -m p \\
-k p & -m p & p(n+1-p)
\end{array}\right], \quad E=\left[\begin{array}{lll}
0 & 1 & 1 \\
1 & 0 & 1 \\
1 & 1 & 0
\end{array}\right] .
$$

We do not, however, adhere to the requirement that $k, m, p$ be integers. Nevertheless, $1 \leqq k \leqq m \leqq p$ is an allowable and useful normalization.

The analogue to the side condition $\varphi=0$ is easily expressed, but in this case there is also a secondary side condition $x E \cdot x=0$, which amounts to $\alpha \beta+\beta \gamma+\alpha \gamma=0$. This all comes about because $\alpha, \beta, \gamma$ were originally three distinct roots to a certain cubic equation ([1], bottom page 105) which had no linear term. As it turns out, neither the relation $\varphi=0$, nor the assumption that $\alpha, \beta, \gamma$ are distinct is required. We simply show $F \geqq(2 / 3)(n+1)$ whenever $x E \cdot x=0$. The beauty of this side condition is that it is independent of the parameters $k, m, p$.

The theory of Lagrange multipliers tells us that for any extremal configuration, there is a nontrivial linear dependence of the gradients, which amounts to $\lambda(x A-v)=\mu x E$. If $\lambda=0$, then $x E=0$, hence $x=0$, and $F=n$. In continuing the investigation, we assume $\lambda=1$.

Next assuming $k=m<p$, the first and second coordinates of $v$ are the same. Therefore the same can be said for $x(A-\mu E)$, and we find easily by subtraction that $[k(n+1)+\mu][\alpha-\beta]=0$, hence either $\alpha=\beta$ or $\mu=-k(n+1)$. With $\alpha=\beta$, the side condition reads $\alpha(\alpha+2 \gamma)=0$, hence either $\alpha=0$ or $\alpha=-2 \gamma$. In the former case $x=(0,0, \gamma)$ and $F=p(2 k+1) \gamma^{2}-2 p \gamma+n$, minimal for $\gamma=1 /(2 k+1)$, with 
value $V_{1}(k, p)=-p /(2 k+1)+n$. In the latter case $x=(-2 \gamma,-2 \gamma, \gamma)$ and $F=$ $\gamma^{2}(8 k+p+18 p k)-2 \gamma(p-4 k)+n, \quad$ minimal for $\gamma=(p-4 k) /(8 k+p+18 p k)$, with value $V_{2}(k, p)=-(p-4 k)^{2} /(8 k+p+18 p k)+n$. The final alternative $\mu=-k(n+1)$ leads (with the side condition) back to $x=(0,0,1 /(2 k+1))$ and $V_{1}(k, p)$. Since both extremal values $V_{1}(k, p)$ and $V_{2}(k, p)$ are less than $n$, we can in future disregard the first extremal configuration $\lambda=0$. We note that $V_{2}(k, p)-V_{1}(k, p)=$ $16 k(p-k)(n+1) /(2 k+1)(8 k+p+18 p k) \geqq 0$, so the minimum for the special case $(k, m, p)=(k, k, n-2 k) \quad$ is $\quad W_{1}(k, n)=V_{1}(k, n-2 k)=2 k(n+1) /(2 k+1)$. We also note $W_{1}(k, n) \geqq(2 / 3)(n+1)$.

We next assume $k<m=p$. By algebraic duality with the previous case, we find the minimum to be $W_{2}(k, n)=V_{2}((n-k) / 2, k)=9 k(n+1)(n-k) /(4 n-3 k+9 k(n-k))$. Finally to interpolate between these cases $p=(n-k) / 2$ and $p=n-2 k$ we observe with $x, k, n$ fixed and $m$ replaced by $n-k-p$, that $F$ is quadratic in $p$ with leading term $-p^{2}(\beta-\gamma)^{2}$. Thus for each fixed $x, k, n$, the minimum of $F$ with respect to $p$ occurs either for $p=n-2 k$ or $p=(n-k) / 2$. It follows that with $k, n$ fixed, $\min \{F(x): x E \cdot x=0, k+m+p=n\}$ is the smaller of the pair $W_{1}(k, n), W_{2}(k, n)$, which happens to be the former. Indeed, one finds

$$
W_{2}(k, n)-W_{1}(k, n)=k(n+1)(n-3 k) /(2 k+1)(4 n-3 k+9 k(n-k)) \geqq 0 .
$$

Since, as observed above, $W_{1}(k, n) \geqq(2 / 3)(n+1)$, we are about done.

Perhaps one should point out that the case $(k, m, p)=(n / 3, n / 3, n / 3)$, strictly speaking not included in the previous analysis, can nevertheless be treated by continuity from the cases considered. It is not surprising and follows from the last formula that $W_{1}(n / 3, n)=W_{2}(n / 3, n)$. Slightly different about this case, however, are the highly nonunique extremal configurations - a locus comprising a space circle.

\section{Reference}

[1] Agard, S., and R. Fehlmann: On the extremality and unique extremality of affine mappings in space. - Ann. Acad. Sci. Fenn. Ser. A I Math. 11, 1986, 87-110.

University of Minnesota

School of Mathematics

Minneapolis, Minnesota 55455

USA

Received 23 March 1987
University of Helsinki

Department of Mathematics

SF-00100 Helsinki

Finland 